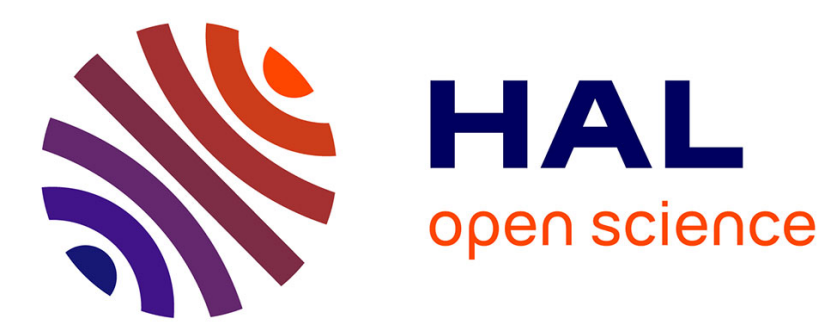

\title{
LES JURÉS À L'ÉPREUVE DES ASSISES : DESCRIPTION ET PORTRAITS D'UNE EXPÉRIENCE MARQUANTE
}

Armelle Jacquemot, Aziz Jellab

\section{> To cite this version:}

Armelle Jacquemot, Aziz Jellab. LES JURÉS À L'ÉPREUVE DES ASSISES : DESCRIPTION ET PORTRAITS D'UNE EXPÉRIENCE MARQUANTE. Les Cahiers de la justice, 2012, 1 (1), pp.31-44. 10.3917/cdlj.1201.0031 . hal-03018476

\section{HAL Id: hal-03018476 https://hal.science/hal-03018476}

Submitted on 22 Nov 2020

HAL is a multi-disciplinary open access archive for the deposit and dissemination of scientific research documents, whether they are published or not. The documents may come from teaching and research institutions in France or abroad, or from public or private research centers.
L'archive ouverte pluridisciplinaire HAL, est destinée au dépôt et à la diffusion de documents scientifiques de niveau recherche, publiés ou non, émanant des établissements d'enseignement et de recherche français ou étrangers, des laboratoires publics ou privés. 


\title{
LES JURÉS À L'ÉPREUVE DES ASSISES : DESCRIPTION ET PORTRAITS D'UNE EXPÉRIENCE MARQUANTE
}

\author{
Armelle Giglio-Jacquemot, Aziz Jellab
}

Dalloz | «es Cahiers de la Justice»

$2012 / 1 N^{\circ} 1 \mid$ pages 31 à 44

ISSN 1958-3702

ISBN 9782996212018

Article disponible en ligne à l'adresse :

https://www.cairn.info/revue-les-cahiers-de-la-justice-2012-1-page-31.htm

Distribution électronique Cairn.info pour Dalloz.

(C) Dalloz. Tous droits réservés pour tous pays.

La reproduction ou représentation de cet article, notamment par photocopie, n'est autorisée que dans les limites des conditions générales d'utilisation du site ou, le cas échéant, des conditions générales de la licence souscrite par votre établissement. Toute autre reproduction ou représentation, en tout ou partie, sous quelque forme et de quelque manière que ce soit, est interdite sauf accord préalable et écrit de l'éditeur, en dehors des cas prévus par la législation en vigueur en France. Il est précisé que son stockage dans une base de données est également interdit. 


\title{
[ ] DOSSIER
}

\section{Les jurés à l'épreuve des assises : description et portraits d'une expérience marquante}

\author{
par Armelle Giglio-Jacquemot et Aziz Jellab
}

\begin{abstract}
Armelle Giglio-Jacquemot, ethnologue, maître de conférences à l'université de Lille 3, chercheuse au CeRIES (centre de recherche Individus, Épreuves, Sociétés), armellej@club-internet.fr.

Aziz Jellab, sociologue, professeur à l'université de Lille 3, chercheur au CeRIES, azizjellab@aol.com

Chaque année en France, quelque 20000 citoyens sont convoqués pour siéger comme jurés d'assises. L'annonce de leur désignation constitue le point de départ d'une expérience marquée par la découverte d'un monde nouveau dans lequel ils doivent construire très vite un rôle auquel ils ne sont guère préparés. Comment ces personnes se représentent-elles par anticipation ce rôle inédit et comment celles qui sont effectivement conduites à l'accomplir vivent-elles leur participation aux assises et ses retombées? C'est à ces questions que l'article se propose de répondre en se penchant sur l'expérience largement méconnue des jurés d'assises.
\end{abstract}

Every year in France, about 20000 citizens have to report for jury duty. The announcement of their designation represents the beginning of an experience marked by the descovery of a new world in which they have to construct a role they are hardly prepared. How they imagine in anticipation this original rôle? And how those which have really accomplished it experienced their participation in the assizes court and its effects? This article pretends to respond to these questions focusing on the widely unknown jurors'experience.

\section{I - Introduction}

Chaque année en France, quelque 20000 citoyens sont convoqués pour siéger comme jurés d'assises ${ }^{1}$ au terme d'une série de tirages au sort dont le premier, effectué à partir des listes électorales, a d'abord eu lieu au niveau de leur commune de résidence. L'annonce de leur désignation représente pour eux le point de départ d'une expé-

1. Beauvallet, Cirendini, 2004. rience marquée par la découverte et l'apprentissage obligés d'un monde nouveau dans lequel ils doivent construire très vite un rôle auquel ils ne sont guère préparés. Comment ces personnes - pour la plupart d'entre elles profanes en matière de droit et de justice pénale - se représentent-elles par anticipation le rôle inédit qu'elles se voient soudainement enjointes à endosser ${ }^{2}$ et comment celles qui sont effectivement

2. La convocation précise que le risque encouru par le juré s'il ne se présente pas au tribunal est une amende de 3750 euros inscrite au casier judiciaire. 
Dossier Les jurés à l'épreuve des assises : description et portraits d'une expérience marquante

conduites à l'accomplir ${ }^{3}$ vivent-elles leur participation aux assises et ses retombées?

C'est à ces questions que nous nous proposons de répondre dans le présent article en nous intéressant d'un peu plus près à l'expérience largement méconnue des jurés d'assises ${ }^{4}$ et en nous appuyant, pour ce faire, sur les matériaux de terrain et les résultats intermédiaires d'une recherche sociologique que nous menons actuellement sur ce sujet ${ }^{5}$.

Nous décrirons cette expérience marquante, généralement vécue comme une épreuve intime et indicible, d'abord sous l'angle de la généralité, avec la mise en évidence de quelques traits caractéristiques, puis sous celui de la singularité, avec les portraits contrastés de deux anciennes jurées qui viendront éclairer la diversité des vécus et la variété des bricolages réalisés par chacun dans la socialisation fulgurante à son nouveau rôle. Car si ces juges d'un jour partagent une commune condition de profane et une commune obligation soudaine de juger et de punir, ces traits très spéci-

3. C'est-à-dire celles qui n'auront pas été dispensées ou encore récusées à l'issue de l'ultime tirage au sort qui préside à la constitution du jury de jugement formé au début de chaque procès.

4. La plupart des publications historiques et sociologiques traitant de la cour d'assises minimisent la place et le rôle des jurés populaires et n'accordent que peu de place à l'expérience socio-subjective de ces acteurs. Si l'on s'interroge sur le caractère démocratique de cette institution (Lombard, 1993; Salas, 2005; Vernier, 2007), sur son statut politique eu égard au mythe du «peuple juge» (Sintomer, 2007; Rosanvallon, 2006), ou encore sur les valeurs morales intervenant dans le jugement (Gruel, 1991), les analyses restent assez générales et évasives sur le travail et les interrogations qui animent les jurés. De leur côté, les recherches ethnographiques se focalisent sur les interactions et les rituels des assises sans s'intéresser à ce que vivent les juges profanes (Jolivet, 2006; Besnier, 2007). fiques ne suffisent pas à en faire des individus semblables face et dans l'expérience. Tous ne passent pas par les mêmes difficultés, les mêmes questionnements ni les mêmes tourments et, quand bien même ils partagent certains d'entre eux, ils ne les vivent pas nécessairement avec la même acuité. Sans compter que le groupe qu'ils forment pour l'occasion, celui des jurés populaires, loin d'être une unité homogène et statique, est en fait constitué de sousensembles qui se forment au long de la session d'assises ${ }^{6}$ sur la base d'affinités et de solidarités qui ne sont pas sans effets sur les vécus individuels.

\section{II - Quelques généralités aux trois moments de l'expérience}

L'expérience par laquelle passent les jurés comporte trois moments, et c'est à tort qu'on considère généralement qu'elle commence et se termine avec la session d'assises parce qu'on identifie sa durée avec

Seuls quelques travaux relevant du champ de la psychologie mettent en relief les épreuves émotionnelles et morales vécues par ces derniers (Scharnitzky et Rainis, 2006 ; Durif-Varembont, 2007).

5. Entamée en 2010, cette recherche a mobilisé des techniques plurielles allant de l'observation de procès à Douai et SaintOmer, en passant par des échanges avec les membres de I'Association des anciens jurés de la cour d'assises du Nord et des entretiens semi-directifs menés dans le Nord-Pas-de-Calais, à Paris et en Basse-Normandie auprès d'une trentaine d'anciens jurés, de dix magistrats et d'une greffière. Nous avons également mobilisé un questionnaire renseigné par une dizaine de magistrats.

6. La cour d'assises se réunit par département généralement tous les trimestres pour une session qui s'étend de deux à trois semaines et comporte plusieurs affaires à juger. Les jurés sont convoqués pour toute la durée d'une session. 
celle de la participation aux procès. Confondre ces deux durées, c'est réduire le temps des jurés à celui de la fonction et du travail que la justice leur fait obligation d'accomplir, alors que l'expérience - avec les épreuves et les interrogations qui la jalonnent - commence pour eux avant la session avec la réception inopinée de leur convocation, et se prolonge au-delà : quelques semaines, des mois, plusieurs années. En d'autres termes, leur travail travaille les jurés avant, pendant et après les assises ${ }^{7}$.

\section{L'avant-session ou le temps de la préparation}

L'annonce de leur désignation - qu'ils apprennent par courrier environ un mois avant la session - marque pour les jurés le début d'une phase de préparation à leur nouveau rôle et à leur plongée dans un monde inconnu. Qu'ils vivent cette désignation comme un honneur ou une malchance, qu'ils l'accueillent avec enthousiasme ou avec appréhension, la convocation aux assises déclenche chez eux un processus d'adaptation par anticipation à ce qui les attend. Ce travail de préparation qu'ils accomplissent en vue de maîtriser au mieux les échéances à venir s'effectue sur trois plans.

Au premier plan des préoccupations qui, chez certains, prennent la forme d'inquiétudes particulièrement vives, il y a la gestion temporelle de la vie familiale et profession- nelle qui implique la mobilisation de divers types de ressources et appuis. C'est à la mise en place d'une autre organisation de leur vie que les jurés travaillent dans la période de l'avant-session, laquelle montre du reste que leur participation n'engage pas qu'eux mais aussi leurs entourages familial, professionnel et amical. Être juré oblige à des réajustements domestiques et professionnels plus ou moins aisés selon les soutiens que l'on peut solliciter, la bonne volonté et la compréhension de son conjoint, de son employeur. Ainsi Bernard, représentant commercial, at-il résolu de prendre sur ses congés pour participer à la session afin d'éviter des ennuis avec son patron. À l'inverse, Éliane, mère de quatre enfants en bas âge, a pu compter sur l'aide de son mari, ses parents, son frère et sa belle-famille pour assurer la continuité d'une organisation familiale déjà compliquée en temps normal. Dans l'avantsession, les jurés œuvrent donc à créer le cadre organisationnel optimal à l'intérieur duquel ils vont pouvoir exercer leur rôle.

Se préparer aux assises c'est aussi, sur un autre plan, s'informer: pour pouvoir justement s'organiser par anticipation mais aussi pour commencer à s'approprier le nouvel univers et le nouveau rôle à investir. Car devenir juré c'est aller vers l'inconnu et l'incertain : «j'allais à l'aventure », disent nombre d'entre eux. Cette quête d'informations motivée par le souci de maîtriser ce qui les attend donne lieu à différentes stratégies selon les préoccupations des uns et des

7. Nombre de jurés utilisent d'ailleurs le terme de travail pour désigner leur activité. 
Dossier Les jurés à l'épreuve des assises : description et portraits d'une expérience marquante

autres. Certains cherchent plutôt des renseignements objectifs sur la cour d'assises et son fonctionnement dans des ouvrages et surtout sur le Net. D'autres, plus inquiets quant aux épreuves qui les attendent, se montrent davantage préoccupés par la dimension subjective de l'expérience et cherchent à accéder à des témoignages d'anciens jurés, que ce soit sur le Net ou

\section{Participer à une session d'assises est une} épreuve pour l'immense majorité des jurés qui sont du reste nombreux à qualifier spontanément l'expérience comme telle.

dans leur entourage. Il en est aussi qui sollicitent des professionnels de la justice et de la psychiatrie dans leurs connaissances ou au hasard des rencontres. La reconnaissance des lieux participe aussi de ce souci de mâ̂triser autant que possible ce qui peut l'être: nombre de jurés se rendent au tribunal avant le début de la session et vont même jusqu'à repérer la salle dans laquelle elle aura lieu. Connaître le chemin, le temps qu'il faudra pour se rendre aux assises passe également par le repérage des possibilités de stationnement à la journée dans les alentours du tribunal.

Enfin, c'est dans cette phase que surgit aussi, pour nombre de jurés, la question de leur légitimité à juger alors qu'ils n'ont pas de compétences juridiques, question qui, pour certains d'entre eux, reste lancinante tout au long de la session et encore au-delà. Les interrogations et les inquiétudes qu'elle suscite travaillent intimement les jurés qui se préparent aussi, en les affrontant, à endos- ser leur nouveau rôle. Le portrait de Christine nous donnera l'occasion de revenir plus amplement sur ce point.

\section{La session ou le temps de l'apprentissage et de l'exercice du rôle}

Participer à une session d'assises est une épreuve pour l'immense majorité des jurés qui sont du reste nombreux à qualifier spontanément l'expérience comme telle.

Depuis les craintes suscitées par la plongée dans l'inconnu d'un monde et d'un rôle qui impressionnent par leur gravité, l'inquiétude suscitée par le sentiment de n'être pas légitime à juger et la peur de ne pas être à la hauteur, en passant par le sentiment d'insécurité que génère la peur d'être reconnu ou retrouvé par les accusés ou leurs proches après le procès; l'angoisse du tirage au sort dont dépend l'intronisation véritable du juré, vivement souhaitée ou redoutée; la violence de la récusation vécue comme une disqualification profondément injuste qui égratigne l'estime de soi ; l'épreuve émotionnelle, mentale, physique et morale que représente la confrontation avec les faits relatés, les auteurs présumés, les victimes et leurs familles, qui montre toute la «cruauté des hommes» comme dit une jurée, épreuve déstabilisante et transformatrice qui fait vaciller les systèmes de valeurs, interroge les préjugés, provoque des dilemmes et des tourments, questionne le sens de la punition et l'équité de la peine, conduit à revoir sa vision de la vie sociale; l'épreuve aussi de la prise de décisions et de la responsabilité vertigineuse qu'implique l'obligation de juger et 
de punir; les souffrances vécues par ceux chez qui les affaires à juger ravivent des événements douloureux et parfois tenus secrets de leur passé (notamment des viols); la solitude éprouvée tout au long d'une expérience qu'on ne peut ou n'arrive pas à partager; l'épreuve enfin, mais nous n'en faisons pas le tour, de la domination, de la manipulation et de la désillusion pour ceux qui sortent de l'expérience désenchantés vis-àvis de la justice pénale, ses pratiques effectives et ses professionnels.

Ces épreuves qui jalonnent la socialisation au monde judiciaire et l'apprentissage comme l'exercice du rôle à y tenir sont diversement rencontrées, vécues et surmontées par les jurés selon, notamment, qu'ils ont été ou non tirés au sort, pour un ou plusieurs procès, selon les crimes qu'ils ont été conduits à juger, selon leur biographie et les soutiens affectifs sur lesquels ils peuvent compter, selon leur appartenance sociale, selon les ressources culturelles et psychiques qu'ils peuvent mobiliser.

Parmi ces épreuves dans l'«épreuve » que représente la participation aux assises, il convient de distinguer celles qui sont transitoires de celles qui sont durables.

Les épreuves transitoires représentent ces étapes qui permettent aux jurés de réaliser la séparation temporaire d'avec leur monde et leurs rôles familiers. Cette coupure est exigée par l'apprentissage du monde et du rôle nouveaux ${ }^{8}$. C'est pourquoi les épreuves qui

8. Cette coupure est rendue tout à fait explicite quand elle est radicale, comme c'est le cas par exemple au Brésil où les jurés sont isolés du monde extérieur pendant la durée des procès. la garantissent se situent au début du processus: elles sont vécues à partir de l'annonce de la désignation et au début de l'immersion dans la session d'assises. Leurs effets (appréhensions, peurs, questionnements, doutes) sont passagers et se dissipent au fur et à mesure que la familiarisation et l'incorporation s'accomplissent. Il s'agit notamment des inquiétudes liées au travail qu'on abandonne, à l'organisation de la vie familiale et quotidienne, à la durée imprévisible des audiences qui ne permettent pas de savoir quand (et même si) on pourra rentrer chez soi le soir, à la peur de l'inconnu. Elles passent au second plan ou deviennent sans objet au cours de la session. On peut considérer ces épreuves comme constitutives du passage entre un monde connu dont on se coupe provisoirement et un monde ignoré auquel on s'intègre en s'agrégeant à un nouveau groupe: de façon temporaire pour la plupart des jurés, plus durablement pour certains d'entre eux qui prolongent leur appartenance à ce nouveau groupe en rejoignant une association d'anciens jurés à l'intérieur de laquelle perdure leur identité de juré audelà de la session ${ }^{9}$.

Les épreuves durables, quant à elles, accompagnent les jurés de bout en bout. Leurs effets peuvent se maintenir ou s'amenuiser au cours de la session comme ils peuvent aussi se prolonger au-delà. Ces épreuves ne sont plus liées, comme les premières, à la nécessaire renonciation à «son »

9. Comme c'est le cas de certains jurés que nous avons interviewés, membres de l'Association des anciens jurés du Nord. 
Dossier Les jurés à l'épreuve des assises : description et portraits d'une expérience marquante

monde et à la plongée dans un autre monde où l'on se sent dénué de tout repère. Directement liées à l'apprentissage et au vécu du rôle de juré lui-même, elles prennent plutôt la forme de doutes, remises en question, dilemmes, tourments, souffrances, perturbations, désillusions, indignations et autres sentiments négatifs tels que l'injustice, le ressentiment et la honte.

\section{L'après-session ou le temps du retour à la vie ordinaire}

Une expérience aussi marquante n'est bien évidemment pas sans effets sur la vie de ceux qui la font et on peut légitimement s'étonner de l'absence générale d'intérêt pour le devenir des jurés aussi bien de la part de la justice que des sciences humaines et sociales. Dans l'ensemble, les jurés se disent «changés» à l'issue des assises. Là encore, on peut distinguer les effets qui se font passagèrement sentir des répercussions plus profondes qui les affectent durablement.

Dans l'immédiate après-session, le retour à la vie normale n'est ni instantané ni facile et les jurés passent par une période de transition qui se traduit par un fort sentiment de «décalage» disent certains, de "perturbation» disent d'autres. Ils se sentent «comme groggy», "dans le coton», «bouleversés». Tout imprégnés de ce qu'ils viennent de vivre, de l'univers et du rôle dans lesquels ils ont dû intensément se couler, des affaires qu'ils ont jugées, des décisions qu'ils ont prises, des personnes qu'ils ont côtoyées - magistrats, jurés, avocats, accusés, victimes - , ils ne «cessent d'y penser» et ont du mal à reprendre contact avec les autres, à réinvestir leur vie, à se concentrer sur leurs tâches, notamment professionnelles. Les jurés retombent dans leur monde plus soudainement encore qu'ils ont dû y renoncer, et ce moment de flottement où l'esprit est ailleurs revêt aussi le caractère d'une épreuve transitoire (non plus d'entrée mais de sortie des assises) vécue et franchie avec plus ou moins de difficultés selon les individus. Ce sentiment de décalage se dissipe avec le temps. Il dure de deux à huit semaines selon les témoignages.

Mais les jurés restent longtemps habités par ce qu'ils ont vécu, qu'ils en témoignent eux-mêmes par des phrases du type "j'y pense toujours. Moins qu'avant mais j'y pense souvent", "encore maintenant je ne m'en remets pas, quand je fais quelque chose, je fais face tout le temps à ça», ou que nous le constations nous-mêmes quand, au cours de nos entretiens, nous percevons leur émotion à l'évocation de leur expérience comme de certaines affaires qu'ils ont eu à juger parfois plusieurs années auparavant. Des jurés qui, pour certains, disent avoir dû entamer une psychothérapie à l'issue de la session.

Car la participation aux assises produit aussi des effets durables et définitifs. "On ne voit plus le monde de la même façon ", disent nombre de jurés et l'image de la «métamorphose», utilisée par certains d'entre eux, figure bien la dimension transformatrice de l'expérience. Les changements sont éprouvés sur divers plans. Les anciens jurés éprouvent un intérêt beaucoup plus vif pour la justice criminelle et son actualité (réformes, procès en cours) qui s'accom- 
pagne parfois du sentiment d'une forme d'expertise dans l'appréciation des procès qui défraient l'actualité. Certains se sentent même investis du rôle d'agir sur le fonctionnement de la justice et de la transformer. Le changement se marque également dans la façon dont ils observent et pensent la société et les comportements humains. Chez certains, l'expérience prend la dimension d'une véritable révélation : «tu vois des choses derrière les choses que tu voyais avant", dit une jurée. La cour d'assises est le théâtre de «drames humains" qui laisse apparaître des faces cachées de la vie en société: elle dévoile des existences, des mondes sociaux et des expériences sociales complexes. Une des épreuves majeures qui travaille les jurés à l'issue des assises renvoie aux prises de conscience et aux chocs générés par la découverte de ce qu'ils appellent «l'horreur», «la violence indescriptible», des «crimes invraisemblables». Cette découverte qui confine aux limites de ce qui est, pour soi, humainement concevable révèle aussi toute la diversité et les possibles de la «nature humaine». Elle impulse une réflexion et une tentative de compréhension des actes dont les hommes sont capables qui ne s'arrêtent pas avec la session; elle interroge et parfois ébranle ses propres valeurs et certitudes; elle change la vision du monde et de la société ou, comme le dit une jurée, la vision de "moi parmi les autres, moi avec les autres, et bien c'est pas si simple». Quant aux changements éprouvés dans le domaine des aptitudes et dispositions personnelles, ils renvoient pour beaucoup au rapport avec les autres: les anciens jurés disent avoir gagné en tolérance et en écoute, en ouverture d'esprit, en maturité, en attention, en capacité de recul et de compréhension, en force de caractère.

\section{III - Les expériences contrastées de deux jurées d'assises}

L'existence de régularités entre les jurés n'empêche pas l'existence de postures particulières et l'on ne saurait prendre toute la mesure de leur expérience sans se centrer sur sa singularité. Cette approche, qui permet d'appréhender toute la variété et la complexité des manières de vivre et de construire cette expérience «extraordinaire», permet aussi de dégager des configurations sociologiquement significatives. Nous nous pencherons sur deux d'entre elles, à travers les portraits de Christine et d'Éliane, choisis ici pour représenter deux vécus très différents d'une expérience dont on pourra ainsi montrer le caractère divers, notamment en ce qui concerne la représentation et la construction du rôle de juré et le rapport aux juges professionnels.

\section{Christine ou l'épreuve de l'illégitimité : entre sentiment d'incompétence et fascination pour le président de la cour}

Écrasée par la responsabilité de juger sans s'en sentir les compétences, Christine (40 ans, directrice d'un centre de formation) est la figure même du juré angoissé, taraudé de bout en bout par des doutes sur sa légitimité et sa capacité à remplir correctement le 
Dossier Les jurés à l'épreuve des assises : description et portraits d'une expérience marquante

rôle qui lui est imposé alors qu'elle est «une personne parmi d'autres», c'est-à-dire «sans formation juridique». Appelée à siéger en 2002 dans les cinq procès que comptait sa session à la cour d'assises du Nord, elle a vécu sa participation comme une «injustice»; et ce sont la difficulté et l'inquiétude qui dominent à l'évocation de son expérience.

Voici comment elle formule les questions qui l'ont assaillie dès la réception de sa convocation :

"Qu'est-ce qu'on attend de moi? Donner mon avis sur les affaires? Je n'y connais rien. Est-ce que je suis la bonne personne pour aller là-bas? Parce qu'on allait nous demander de jouer un rôle, d'avoir une fonction que je ne maitrisais absolument pas, que je n'imaginais absolument pas. Pourquoi on est là, qu'est-ce qu'on est censé faire là, quelle légitimité on a à participer à un jury d'assises? Pourquoi, moi, je devrais juger quelqu'un, en vertu de quoi? »

À l'approche et au début de la session, elle a ressenti une forte anxiété augmentée d'un pénible sentiment de coercition, jamais éprouvé jusque-là : «je voulais partir [de la salle d'audience] et je ne pouvais pas», ditelle à diverses reprises. Pour faire face à la situation, elle a d'abord cherché à empoigner rationnellement son rôle en tentant d'identifier des critères de jugement qui lui permettent d'être juste. Car, dans la conception qu'elle s'en fait, pour être légitime à juger quand on n'en a ni les compétences ni la légitimité juridiques, il faut être juste. Et pour cela, il faut disposer de critères objectifs de jugement. C'est dans ce but qu'elle a commencé par élaborer «des grilles d'évaluation» :

"Je me suis dit qu'il fallait arriver à trouver des critères qui me permettent d'être le plus juste possible, avoir un système qui permette de se raccrocher, d'être logique. Donc j'avais fait une grille avec les circonstances, s'il avait une arme ou pas, des coups ou pas, s'il était seul ou pas, il y avait tout un tas d'items que j'avais sélectionnés... C'est-à-dire qu'avec ma grille je pensais que j'allais m'en sortir. J'avais hiérarchisé. Pour la première affaire, admettons que ça ait marché (...) À la deuxième affaire, j'ai voulu reprendre ma grille, mais étrangement elle ne m'aidait pas à évaluer la gravité du crime... c'était différent. Les cases que j'avais remplies, elles ne m'apportaient rien pour faire une évaluation, une comparaison avec l'affaire précédente. J'arrivais pas à dire "c'est plus grave", "c'est moins grave" parce que c'était différent. Donc, j'ai fait une autre grille, en essayant d'ajouter des aspects plus... du domaine $d u$ ressenti; "ça me paraît plus grave, pourquoi?», "comment est-ce que je le ressens, pourquoi? ", avec des «+ + , des «--», enfin une notation. Est arrivée la troisième affaire, et, là, j'ai décidé de jeter mes grilles parce que je pouvais encore ouvrir une autre grille, ça ne m'apportait rien »

Après cette tentative qui, à l'expérience de juger, s'est révélée vaine, Christine s'est abandonnée à l'exercice - "j'ai décidé de travailler sans filet » - et parle d' «improvisation» pour désigner la façon dont elle s'y est finalement prise pour jouer son rôle du mieux possible.

Tendue vers un but ultime, "prendre une décision la plus juste possible», son improvisation a été guidée par des exigences morales fortes : «être le plus honnête, le plus juste pos- 
sible», et des principes gouvernant la bonne attitude à adopter : "être le plus sérieux possible», "garder cette capacité à être disponible pour écouter les choses", "se transcender pour être vraiment bien », "avoir pour chaque affaire un état d'esprit juste pour ne pas mélanger, ne pas saturer». S'efforcer d'être le plus juste possible pour l'autre que l'on juge, certes, mais également pour soi-même: il s'agit en effet d'en sortir avec la conscience tranquille et de pouvoir laisser le juge que l'on a été et les décisions que l'on a prises derrière soi.

Néanmoins, ne s'en remettre qu'à ellemême pour juger et décider de la peine a généré chez Christine une inquiétude constante que seule est venue atténuer la figure centrale et impressionnante d'un président «admirable» et rassurant qui a d'ailleurs éclipsé celle, insignifiante, des assesseurs qui ne l'ont pas aidée. À ses côtés, il a joué un rôle fondamental tout au long de l'expérience parce qu'il a représenté la seule référence solide à laquelle elle a pu se «raccrocher». Car si Christine avait pour elle ses exigences et ses principes, ils ont été impuissants à produire par eux-mêmes une capacité de discernement qui lui permette d'être assurée de bien faire et donc de "prendre la décision la plus juste possible». Celui qui a fourni les éléments de clairvoyance qui lui manquaient, ce fut le président; et quand Christine parle de «l'aide» précieuse qu'il lui a apportée, comme aux autres jurés, c'est en cela qu'elle a consisté.

Ce président dans lequel elle a eu une confiance totale, Christine le compare à «un père», "une maman», "une lumière », «un point de repère». Il incarne l'autorité et la protection d'un parent d'expérience qui veille avec bienveillance sur les apprentissages de ses enfants :

«J'ai vraiment senti... un peu comme un enfant qui apprend à marcher. Tu es autour de lui sans le toucher, tu le laisses. Ou alors un enfant qui apprend à faire du vélo. Tu tiens délicatement sa selle à l'arrière. Il est autour de nous... si tu tombes, il te retient».

Le président apparaît comme la référence à partir de laquelle elle a construit ses décisions. Pour autant, Christine tient spontanément à souligner qu'elle n'a pas été

\section{S'efforcer d'être le plus juste possible pour l'autre que l'on juge, certes, mais également pour soi-même : il s'agit en effet d'en sortir avec la conscience tranquille.}

influencée par ce dernier alors que ses propos témoignent clairement du contraire. C'est que l'influence du président est pour elle si légitime que ce magistrat ne lui est pas apparu comme une source d'influence.

Sa participation aux assises aura donc été très difficile pour Christine - «une expérience dure que je ne souhaite pas revivre » - et la narration qu'elle en fait met en évidence de nombreuses autres épreuves au côté de celle, centrale, que nous venons de décrire. De cette expérience qu'elle qualifie à la fois d'«épreuve » et d' «apprentissage », cette jurée a tiré un ensemble d'enseignements et assimile à une «métamorphose " les transformations qu'elle a engendrées : «ça modifie notre façon d'envisager ce que nous sommes et notre rapport avec les autres", dit-elle. Une rupture s'est produite entre un avant - où elle s'est 
Dossier Les jurés à l'épreuve des assises : description et portraits d'une expérience marquante

aperçue qu'elle était aveugle à ce qui l'entourait - et un après - où sa vision de la société, d'autrui et du vivre ensemble s'est comme amplifiée et complexifiée : «cette ouverture vers autre chose, elle est difficile parce que tu ne lui refermes pas la porte après ». Ce que Christine a gagné aux assises, c'est un regard étendu sur ce qui existe au-delà de ce qui la concerne directement. Comme si elle avait pris acte de la diversité de ce qu'elle appelle «la nature humaine », des opinions et des modes d'être dans le monde, et de la dimension collective de la vie en société.

Et si elle dit être désormais "davantage sensible aux choses et aux gens », si elle affirme qu'être jurée lui a permis de ne "pas continuer à viure en regardant à travers un rouleau en ignorant pas mal de choses ", si elle témoigne du fait que «ça montre que vivre ensemble, c'est forcément compliqué, ça ne va pas de soi, c'est un travail collectif», elle estime néanmoins que "c'est une expérience dont on pourrait se passer, on vivrait tout aussi bien ».

\section{2. Éliane ou l'épreuve de la domination : quand l'enthousiasme cède au désenchantement}

Appelée à siéger en 2008 dans deux des procès que comptait sa session à la cour d'assises du Nord, Éliane (35 ans, secrétaire médicale) est la figure même du juré déçu et acerbe. Face aux pratiques judiciaires effectives et aux rapports de pouvoir instaurés entre jurés et magistrats, son enthousiasme premier a cédé peu à peu la place à l'amertume et au désenchantement vis-à-vis d'une justice perçue comme injuste et contestable. Ce sont l'indignation et la désillusion qui dominent à l'évocation de son expérience dont l'épreuve centrale est celle de la domination et de la manipulation par les juges professionnels.

Pourtant, à la différence de Christine, Éliane se rappelle avoir éprouvé un grand contentement à la réception de sa convocation. Elle l'a vécue comme «un honneur» qui s'est accompagné du sentiment d'être reconnue, d'avoir «presque été choisie». Le caractère incertain de l'expérience ne l'a pas effrayée: elle l'a envisagée positivement «comme une aventure " et l'a appréhendée sereinement "en laissant venir les choses". Elle désirait ardemment investir le rôle qui lui était dévolu et sa désignation comme sa participation ne semblent pas avoir suscité de questionnements sur sa légitimité à juger. À l'image de Christine, Éliane se définit pourtant comme "une personne lambda». Mais loin d'avoir représenté un problème, sa méconnaissance du droit lui est au contraire apparue comme au fondement même de sa légitimité. Elle a été désignée comme jurée non pour ses compétences juridiques mais pour juger avec ce qu'elle « $a »:$ «on va juger avec nos émotions, avec notre expérience, on n'a que ça, nous, pour juger ». Sa condition de juge profane ne l'a donc pas inquiétée et lorsqu'elle a été tirée au sort pour la première fois : «j'étais contente», dit-elle, «je me suis dit: "enfin, je vais pouvoir remplir mon rôle" ».

À la différence de Christine, le discours qu'elle déploie durant l'entretien n'est pas axé sur elle, ses difficultés, ses peurs, sa 
transformation. Il est orienté sur le fonctionnement des assises et le rôle des juges, magistrats et jurés. Pour autant, cette expérience abordée avec confiance et enthousiasme a aussi revêtu pour elle le caractère d'une rude épreuve au terme de laquelle la justice a perdu sa légitimité.

Tout d'abord, des deux procès auxquels elle a participé, l'un lui a semblé «injuste» quant au verdict, et l'autre "pas correct» quant à la façon dont il a été mené. Du second procès, une affaire de viol, elle dit:

«Il y avait beaucoup trop de zones d'ombre, le procès partait un peu dans tous les sens et, $d u$ coup, j'étais des fois en colère de la façon dont il se déroulait (...) Il était trop léger au niveau des preuves et, de toute façon, je trouvais que le présumé innocent arrivait plus comme un présumé coupable (...) donc on allait arriver à le condamner quoi qu'il en soit. J'ai pas aimé».

Ensuite, elle pose sur les jurés un regard très différent de celui de Christine: si cette dernière les dépeint responsables et soucieux de faire de leur mieux, Éliane en fait quant à elle un portrait symétriquement inversé. Beaucoup sont intellectuellement faibles, indifférents et inconsistants, sans opinion propre, à la recherche du consensus. En d'autres termes, «complètement manipulables » et «manipulés» par les juges professionnels.

Elle ne partage pas non plus avec Christine sa vision admirative du président de la cour et son sentiment de reconnaissance pour l'aide apportée dans l'épreuve. Ce que l'une a vécu comme un accompagnement précieux, l'autre l'a vécu comme une manipulation permanente garantie par «le fait qu'on soit mêlés à la magistrature constamment». Chez les juges professionnels, Éliane a surtout perçu le rapport de domination sur fond de pratiques destinées à garantir leur pouvoir sur les juges profanes. Elle a fait l'expérience révoltante d'un rapport de force très défavorable aux jurés :

«On est face à des hommes de droit qui connaissent les lois. Nous, on n'est rien, on est lambda. Je pense que le rôle de juré d'assises est très minoré par rapport à... ces trois personnes devant toi ${ }^{10}$ qui pendant trois jours vont te faire comprendre... tu comprends ce qu'ils veulent te dire. C'est frappant. Donc je pense qu'il n'y a pas d'impartialité finalement. Ça m'a beaucoup dérangée... même si on doit juger au même titre qu'eux, on n'a finalement pas le même rôle, il n'y a rien à faire. Ils ont une robe, on vient en jean-baskets... les rôles sont bien définis à la base. Par exemple, on n'a jamais déjeuné ensemble ; eux, ils s'incrustaient dans notre salle de jurés mais nous, on ne s'incrustait pas dans leur bureau. On aurait pu s'incruster dans leur bureau, dire "ben tiens, on vient boire le café chez vous pour une fois, de toute façon on va juger la même affaire». On ne se serait pas permis. Pourquoi on s'est pas permis ça? Parce que déjà c'était verrouillé chez eux. Nous, on n'a pas verrouillé. Mais eux, ils verrouillent automatiquement...».

La domination et la manipulation des jurés, Éliane les a identifiées dans divers moments et pratiques qu'elle n'a «pas aimés » et qui l'ont «beaucoup dérangée ».

10. Le président de la cour et ses deux assesseurs. 
Dossier Les jurés à l'épreuve des assises : description et portraits d'une expérience marquante

C'est notamment le cas des récusations qui ponctuent le tirage au sort du jury de jugement. Elles lui sont apparues comme une pratique manipulatoire qui permet d'arranger le jury selon les stratégies des uns et des autres et qui, par suite, fausse le procès. Le groupe des jurés n'est pas le fruit du sort pourtant censé être au fondement même de sa constitution et garantir son caractère démocratique. C'est ce constat qui l'a déçue et qui la révolte. Elle est même convaincue que le président de la cour donne des consignes aux avocats pour écarter certains jurés, notamment ceux qui sont devenus trop experts pour avoir participé à plusieurs procès car «ils dérangent». En contact permanent avec les jurés, c'est lui en effet qui les connaît le mieux, qui les soupèse et les sonde au moyen des multiples échanges qu'il a avec eux.

Le délibéré marque quant à lui l'apogée de la manipulation puisqu'il ne sert qu'à emporter un vote des jurés conforme à ce que souhaitent les magistrats : «c'est le point de chute", dit Éliane. Les discussions engagées et contrôlées par le président lui sont apparues comme un simulacre de démocratie dans lequel les jurés ne sont qu'un alibi :

"Quand le procès est terminé et qu'on se retrouve les neuf jurés et les magistrats pour faire ce fameux délibéré, j'ai du mal à comprendre pourquoi on passe une, deux, trois, quatre heures à délibérer alors qu'on nous demande de voter avec notre intime conviction. C'est que, quelque part, ils ont envie que notre intime conviction dévie vers leur propre conviction à eux, les magistrats. Sinon, quel est l'intérêt d'attendre trois heures pour délibérer. Le procès on l'a vu. Ils refont le procès une deuxième fois dans un huit clos pour amener le juré... j'ai l'impression qu'ils raisonnent le juré, qu'ils l'amènent vers ce que le magistrat veut ».

À la fin de son deuxième et dernier procès, Éliane s'est affranchie de la domination exercée par les magistrats grâce à un acte de «rébellion». Elle a osé affirmer sa propre conviction dans un vote contestataire:

«J'allais voter blanc au départ. Parce que j'assumais pas. Et puis, au dernier moment, je me suis dit : "faut que j'assume aussi, je suis là pour ça ". Donc j'étais finalement contente d'avoir assumé ce que moi je pensais vraiment. Et ne pas toujours être dans le consensus des plus forts. Je sentais qu'on attendait ça de nous, qu'on le condamne. Ah oui, complètement (...) Moi, je n'étais pas d'accord avec ça, et peut-être que mon vote a été une sorte de rébellion mais avec une conviction quand même, pas genre "je vais faire l'inverse de ce qu'il a dit », j'ai beaucoup réfléchi. Mais voilà, j'ai contesté de manière assez radicale ».

Son expérience de jurée l'a laissée si amère que, à la différence de Christine, elle estime qu'elle ne lui a rien apporté : «dans ma vie, ça n'a rien changé», dit-elle. Au terme de l'aventure, l'orgueil et l'enthousiasme suscités par sa convocation paraissent comme l'envers d'une expérience marquée par le désenchantement et le ressentiment. Le fonctionnement de la cour d'assises et les stratégies des magistrats ne se sont pas révélés dignes de l'effort qu'elle a engagé pour tenir son rôle. Aux assises, Éliane a perdu sa vision magnifiée du monde de la justice. 


\section{IV - Conclusion}

Les expériences contrastées de Christine et d'Éliane n'épuisent pas la pluralité des vécus et, si ces deux anciennes jurées ne souhaitent pas de nouveau remplir ce rôle, il en est d'autres qui, «déçus que ça s'arrête », auraient volontiers poursuivi leur travail audelà de la session et espèrent une nouvelle convocation, d'autant plus opportune à leurs yeux qu'ils se sentent désormais expérimentés. Les jurés oscillent entre enthousiasme et déception, confiance et inquiétude, déférence vis-à-vis des magistrats et dénonciation de leur emprise, sentiment d'avoir été importants et sensation de n'avoir pas pesé. Pour tous néanmoins, les assises représentent un monde d'épreuves au sortir duquel ils ne sont plus les mêmes. Expérience marquante, la participation au jury de jugement ouvre les jurés sur le monde social, sa cruauté et sa complexité, et les conduit souvent à penser autrement la vie sociale; elle leur apprend l'exercice démocratique qui, malgré ses limites et ses contradictions ${ }^{11}$, apporte une reconnaissance, notamment aux citoyens les plus modestes socialement; elle les questionne et les éclaire sur eux-mêmes (notamment sur leurs pré- jugés et leurs valeurs à travers l'épreuve d'un jugement autrement plus engageant que lorsqu'il s'agit de juger de manière générale les faits sociaux ou les faits divers). Expérience morale s'il en est, la participation aux assises est aussi une expérience citoyenne dont notre enquête souligne les effets plus ou moins durables. Dans leur majorité, les anciens jurés se sentent plus concernés par les affaires publiques. Et si certains cherchent à laisser derrière eux cette parenthèse dans leur vie, d'autres au contraire s'appliquent à

Expérience morale s'il en est, la participation aux assises est aussi une expérience citoyenne dont notre enquête souligne les effets plus ou moins durables.

la prolonger en s'engageant dans des associations (d'anciens jurés, humanitaires, pour la lecture en prison,...) qui leur laissent entrevoir la possibilité d'agir et de peser sur la justice ou plus largement sur la société. Comme si le fait d'avoir été institués juges et d'avoir accompli ce rôle engendrait chez ces citoyens plus tout à fait ordinaires le sentiment d'une compétence politique légitime et une volonté d'implication dans la vie de la cité.
11. Par exemple, les jurés sont invités à poser des questions pendant les audiences mais ils sont en même temps dissuadés de les formuler directement sous prétexte qu'ils risquent de dévoiler leur opinion et donc d'être récusés. 
Dossier Les jurés à l'épreuve des assises : description et portraits d'une expérience marquante

\section{Bibliographie}

C. Beauvallet, O. Cirendini, Cour d'assises. Et si demain vous étiez juré? Paris, Jalan Publications, 2004.

C. Besnier, «La cour d'assises. Approche ethnologique du judiciaire », Droit et Cultures, 54/2, 2007, p. $179-202$.

J. P. Durif-Varembont, «La souffrance psychique des jurés de cour d'assises et les modalités de son traitement », Bulletin de psychologie, 60 (5), 491, 2007, p. 441-446.

L. Gruel, Pardons et châtiments, Paris, Nathan, 1991.

A. Jolivet, «Juré en cour d'assises : découverte d'un monde social et expérience de sociabilité au sein d'un groupe restreint », Droit et société, no 62/1, 2006, p. 203-222.

F. Lombard, Les jurés. Justice représentative et représentations de la justice, Paris, L'Harmattan, 1993.

P. Rosanvallon, La contre-démocratie. La politique à l'âge de la défiance, Paris, Seuil, 2006.

D. Salas, La volonté de punir. Essai sur le populisme pénal, Paris, Hachette Littératures, 2005.

P. Scharnitzky, N. Rainis, «Juré d'assises : une expérience de citoyenneté », Le journal des psychologues, $\mathrm{n}^{\circ}$ 241, 2006, p. 32-36.

Y. Sintomer, Le pouvoir au peuple. Jurys citoyens, tirage au sort et démocratie participative, Paris, La Découverte, 2007.

D. Vernier, Jury et démocratie : une liaison fructueuse? L'exemple de la cour d'assises française, thèse ENS Cachan, 2007. 